\title{
Similarity and differences in small dense low-density lipoprotein assessment: two methods compared
}

Kazuhiko Kotani ${ }^{1}$, Naoki Sakane ${ }^{2}$, Alejandro Gugliucci ${ }^{3}$

${ }^{1}$ Division of Community and Family Medicine, Jichi Medical University, Shimotsuke-City, Japan

${ }^{2}$ Division of Preventive Medicine, National Hospital Organization Kyoto Medical Center, Kyoto-City, Japan

${ }^{3}$ Glycation, Oxidation and Disease Laboratory, Touro University, California, CA, USA

Submitted: 26 June 2021

Accepted: 4 August 2021

Arch Med Sci Atheroscler Dis 2021; 6: e166-e168

DOI: https://doi.org/10.5114/amsad.2021.109257

Copyright (c) 2021 Termedia \& Banach

Laboratory tests of lipoproteins/lipids, such as low-density lipoprotein $(\mathrm{LDL})$ cholesterol (LDL-C), triglycerides (TG) and high-density lipoprotein cholesterol (HDL-C), are widely applied in clinical practice. LDL-C is an established marker of cardiovascular disease (CVD) risk; however, as the LDL particles are known to be heterogeneous in terms of size, density and composition, the different particles can exhibit different involvement in CVD [1, 2]. A reduction of LDL size is caused by overproduction of hepatic very low-density lipoproteins and decreased clearance of circulating LDL in some conditions (e.g., insulin resistance, hypertriglyceridemia, glucose intolerance, metabolic syndrome) [2, 3]. The resulting small dense LDL (sdLDL) particles are more atherogenic due to their high susceptibility to oxidation and glycation, high penetration of arterial intima, and prolonged residence in blood circulation due to low affinity for the LDL receptor [4]. SdLDL is currently recognized as a potentially causative lipoprotein particle for CVD $[1,2]$.

There exist various methods to measure sdLDL, for instance, using density gradient ultracentrifugation, gel electrophoresis, high-performance gel filtration chromatography and nuclear magnetic resonance [5]. These methods are expensive and unavailable for routine clinical practice. Moreover, data on SdLDL are not necessarily comparable across these methods based on the different methodological principles and even definitions of sdLDL [5-7]. Recently, a technique to measure sdLDL cholesterol (sdLDL-C) has been developed using a homogeneous direct method that dissociates sdLDL particles enzymatically (Denka Seiken, Co. Ltd., Tokyo, Japan) [8]. It is of concern to compare sdLDL-C with other methods approved for clinical laboratory use. One such method, the Lipoprint system for LDL-subfractional analysis (Quantimetrix Corp., CA, USA), is a standardized method using polyacrylamide gel electrophoresis to stain all lipids, which provides information of the sdLDL relative percentage as well as average LDL size [6,7]. Here, we aimed to compare these two methods.

The current study investigated 38 asymptomatic subjects (mean age $=57.2$ years; men/women $=4 / 34$ ). We excluded subjects with a history of diabetes mellitus and coronary heart disease as well as the use of anti-hypertensive, anti-diabetic and cholesterol-lowering drugs. The Ethics Committee approved the study (No. K15-033, J20-038), and the subjects gave their informed consent. The mean blood pressure (BP) was calcu-

\author{
Corresponding author: \\ Prof. Kazuhiko Kotani \\ Division of Community \\ and Family Medicine \\ Jichi Medical University \\ 3311-1 Yakushiji \\ 329-0498 Shimotsuke-City \\ Japan \\ Phone: +81285 587394 \\ E-mail: kazukotani@jichi.ac.jp
}


Table I. Clinical data of the studied subjects

\begin{tabular}{|lcccc|}
\hline Variable & Levels & sdLDL-C & sdLDL percentage & LDL size \\
\hline BMI $\left[\mathrm{kg} / \mathrm{m}^{2}\right]$ & $24.4 \pm 2.4$ & $0.12(0.46)$ & $0.05(0.75)$ & $-0.09(0.57)$ \\
\hline Mean BP $[\mathrm{mm} \mathrm{Hg}]$ & $100 \pm 13$ & $0.14(0.41)$ & $0.78(0.64)$ & $-0.37\left(0.02^{*}\right)$ \\
\hline Glucose $[\mathrm{mg} / \mathrm{dl}]$ & $94 \pm 9$ & $-0.12(0.49)$ & $0.11(0.53)$ & $-0.13(0.43)$ \\
\hline LDL-C $[\mathrm{mg} / \mathrm{dl}]$ & $141 \pm 36$ & $0.74\left(<0.01^{\star}\right)$ & $0.32(0.05)$ & $-0.38\left(0.02^{\star}\right)$ \\
\hline HDL-C $[\mathrm{mg} / \mathrm{dl}]$ & $67 \pm 20$ & $0.00(0.99)$ & $-0.34\left(0.04^{\star}\right)$ & $0.32(0.05)$ \\
\hline Triglycerides $[\mathrm{mg} / \mathrm{dl}]$ & $103(76-144)$ & $0.69\left(<0.01^{\star}\right)$ & $0.24(0.15)$ & $-0.56\left(<0.01^{\star}\right)$ \\
\hline sdLDL-C $[\mathrm{mg} / \mathrm{dl}]$ & $31(20-52)$ & - & $0.35\left(0.03^{*}\right)$ & $-0.62\left(<0.01^{\star}\right)$ \\
\hline sdLDL percentage $(\%)$ & $13.1(3.2-20.8)$ & $0.35\left(0.03^{*}\right)$ & - & $-0.79\left(<0.01^{\star}\right)$ \\
\hline LDL size $[\AA]$ & $263.2 \pm 7.0$ & $-0.62\left(<0.01^{\star}\right)$ & $-0.79\left(<0.01^{\star}\right)$ & - \\
\hline
\end{tabular}

$B M I$ - body mass index, $B P$ - blood pressure, $L D L$ - low-density lipoprotein, $L D L-C$ - LDL cholesterol, HDL-C - high-density lipoprotein cholesterol, sdLDL - small dense $L D L$, sdLDL-C - small dense $L D L$ cholesterol. The levels of each variable are shown as the mean \pm standard deviation or median (interquartile range). The correlation between each variable and $s d L D L-C$, sdLDL percentage or $L D L$ size is shown as the $r$ coefficient (p-value). Triglycerides, sdLDL-C and sdLDL percentage were log-transformed because of their skewed deviation. *Significance: $p<0.05$.

lated based on the systolic/diastolic BP measured in the right upper arm of the seated subject using a standard sphygmomanometer. In fasted blood samples, lipid and glucose levels were enzymatically measured. The LDL-C levels were measured by a direct assay. We measured the SdLDL-C levels using an enzymatic assay [8] and the sdLDL percentage (\%) and LDL size ( $\AA$ ) using the Lipoprint system [7]. We analyzed the correlations between the variables. Triglycerides, sdLDL-C and sdLDL percentage were used after log-transformation because of their skewed distribution. A $p<0.05$ was considered significant.

Table I shows the clinical data of this population. With higher sdLDL-C levels, the sdLDL percentage was higher $(r=0.35)$ and the LDL size was smaller $(r=-0.62)$. The sdLDL-C levels were significantly positively correlated with the LDL-C $(r=0.74)$ and TG levels $(r=0.69)$. The sdLDL percentage was marginally positively correlated with the LDL-C $(r=0.32)$ and significantly inversely with HDL-C levels $(r=-0.34)$. There was a significant inverse correlation between the sdLDL percentage and LDL size $(r=-0.79)$. The LDL size was significantly inversely correlated with LDL-C $(r=-0.38)$ and TG levels $(r=-0.56)$. Additionally, the LDL size was significantly inversely correlated with mean BP $(r=-0.37)$.

The current study found a significant, but not always a close, relationship among the two methods, the sdLDL-C and Lipoprint markers. This finding seemed reasonable due to the different approach as enzymatic versus size-based methods [7, 8]. Of note, the positive correlation of SdLDL-C with LDL-C was clear relative to the other markers, which is in line with earlier studies $[9,10]$. Their clearly high correlation might be due to the fact that while Lipoprint captures all lipids of lipoproteins, both sdLDL-C and LDL-C measure directly cholesterol content in lipoproteins. Indeed, by their high correlation, the predictive value of sdLDL-C is attenuated in a simultaneous consideration of LDL-C on CVD outcomes $[9,10]$. Additionally, unlike with the other markers, an inverse correlation of LDL size with BP levels was also of note. While the reasons for their correlation were unclear, the LDL size may be a sensitive marker of systemic endothelial and circulatory conditions [3].

This preliminary study has certain limitations. The studied population was relatively small. The cross-sectional design did not determine causality. The statistical analyses used in this study do not address between-marker concordance but correlations only.

In conclusion, there were significant, albeit not always tight correlations between sdLDL-C measured by an enzymatic direct method and sdLDL percentage as well as LDL size measured by Lipoprint. This suggests that the respective methods for sdLDL assessment may be relevant for CVD risk with advantages and disadvantages.

\section{Acknowledgments}

This work was partly supported by a MEXT KAKENHI Grant (No. JP 19K07872).

\section{Conflict of interest}

The authors declare no conflict of interest.

\section{References}

1. Rizzo M, Berneis K. Low-density lipoprotein size and cardiovascular prevention. Eur J Intern Med 2006; 17: 77-80.

2. Gerber PA, Nikolic D, Rizzo M. Small, dense LDL: an update. Curr Opin Cardiol 2017; 32: 454-9.

3. Ikeda K, Suzuki M, Ikebuchi M, et al. Hyperbetalipoproteinemia with small low-density lipoprotein, a characteristic disorder of lipoprotein in essential hypertension. J Diabetes Complications 1995; 9: 227-9.

4. Chancharme L, Thérond P, Nigon F, et al Cholesteryl ester hydroperoxide lability is a key feature of the oxidative 
susceptibility of small, dense LDL. Arterioscler Thromb Vasc Biol 1999; 19: 810-20.

5. Chung M, Lichtenstein AH, Ip S, Lau J, Balk EM. Comparability of methods for LDL subfraction determination: a systematic review. Atherosclerosis 2009; 205: 342-8.

6. Varady KA, Lamarche B. Lipoprint adequately estimates LDL size distribution, but not absolute size, versus polyacrylamide gradient gel electrophoresis. Lipids 2011; 46: 1163-7.

7. Hoefner DM, Hodel SD, O'Brien JF, et al. Development of a rapid, quantitative method for LDL subfractionation with use of the Quantimetrix Lipoprint LDL System. Clin Chem 2001; 47: 266-74.

8. Ito Y, Fujimura M, Ohta M, et al. Development of a homogeneous assay for measurement of small dense LDL cholesterol. Clin Chem 2011; 57: 57-65.

9. Hoogeveen RC, Gaubatz JW, Sun W, et al. Small dense low-density lipoprotein-cholesterol concentrations predict risk for coronary heart disease: the Atherosclerosis Risk In Communities (ARIC) study. Arterioscler Thromb Vasc Biol 2014; 34: 1069-77.

10. Arai H, Kokubo $Y$, Watanabe $M$, et al. Small dense low-density lipoproteins cholesterol can predict incident cardiovascular disease in an urban Japanese cohort: the Suita study. J Atheroscler Thromb 2013; 20: 195-203. 